\title{
Mitochondrial dysfunction results from oxidative stress in the skeletal muscle of diet-induced insulin-resistant mice
}

\author{
Charlotte Bonnard, ,1,2,3,4,5 Annie Durand, ,1,2,3,4,5 Simone Peyrol, ${ }^{6}$ Emilie Chanseaume, ${ }^{7}$ \\ Marie-Agnes Chauvin, ${ }^{1,2,3,4,5}$ Béatrice Morio, ${ }^{7}$ Hubert Vidal, ${ }^{1,2,3,4,5}$ and Jennifer Rieusset ${ }^{1,2,3,4,5}$ \\ ${ }^{1}$ INSERM, U870, IFR62, Oullins, France. ${ }^{2}$ INRA, UMR1235, Oullins, France. ${ }^{3 I N S A-L y o n, ~ R M N D, ~ V i l l e u r b a n n e, ~ F r a n c e . ~}$ \\ ${ }^{4}$ Université Lyon 1, Lyon, France. ${ }^{5}$ Hospices Civils de Lyon, Lyon, France. 6 FIFR62, Centre Commun d'Imagerie de Laennec, \\ Université Lyon 1, Lyon, France. ${ }^{7}$ INRA, UMR 1019, Clermont-Ferrand, France.
}

\begin{abstract}
Mitochondrial dysfunction in skeletal muscle has been implicated in the development of type 2 diabetes. However, whether these changes are a cause or a consequence of insulin resistance is not clear. We investigated the structure and function of muscle mitochondria during the development of insulin resistance and progression to diabetes in mice fed a high-fat, high-sucrose diet. Although 1 month of high-fat, high-sucrose diet feeding was sufficient to induce glucose intolerance, mice showed no evidence of mitochondrial dysfunction at this stage. However, an extended diet intervention induced a diabetic state in which we observed altered mitochondrial biogenesis, structure, and function in muscle tissue. We assessed the role of oxidative stress in the development of these mitochondrial abnormalities and found that diet-induced diabetic mice had an increase in ROS production in skeletal muscle. In addition, ROS production was associated with mitochondrial alterations in the muscle of hyperglycemic streptozotocin-treated mice, and normalization of glycemia or antioxidant treatment decreased muscle ROS production and restored mitochondrial integrity. Glucose- or lipid-induced ROS production resulted in mitochondrial alterations in muscle cells in vitro, and these effects were blocked by antioxidant treatment. These data suggest that mitochondrial alterations do not precede the onset of insulin resistance and result from increased ROS production in muscle in diet-induced diabetic mice.
\end{abstract}

\section{Introduction}

The prevalence of type 2 diabetes increases dramatically in modern societies, in part because of ample food supplies and a sedentary lifestyle. Excess dietary fat and sugar plays a crucial role and are determinants of the current epidemic. In peripheral tissues, increased flux of energy fuel substrates associated with such diets leads to ectopic lipid accumulation, generation of ROS, and cellular dysfunction, referred to as gluco-lipo-toxicity (1).

Over the past few years, an increasing number of studies has linked lipid accumulation in skeletal muscle to reduced insulin sensitivity in various groups of subjects, including type 2 diabetic patients (2-4). In addition, intracellular lipid metabolites, such as fatty acyl-CoA, diacylglycerol, or ceramide, have been shown to inhibit insulin action (5) via activation of serine/threonine kinases and serine phosphorylation of IRS1 (6). Both increased fatty acid uptake and decreased fatty acid oxidation may induce lipid accumulation in skeletal muscle. Studies in humans (7) and rodents (8) have demonstrated that increased fatty acid uptake into muscle contributes to lipid accumulation in situations of insulin resistance. In addition, there is growing evidence that mitochondrial dysfunction in skeletal muscle, and the subsequent impaired ability to

Nonstandard abbreviations used: COX, cytochrome $c$ oxidase; CS, citrate synthase; ERR $\alpha$, estrogen-related receptor $\alpha$; HFHSD, high-fat, high-sucrose diet; HPRT, hypoxanthine guanine phosphoribosyl transferase; Mfn2, mitofusin 2; mtDNA, mitochondrial DNA; mtTFA, mitochondrial transcription factor; NAC, N-acetylcysteine; NBT, nitroblue tetrazolium; NRF, nuclear respiratory factor; PGC1, PPAR $\gamma$ coactivator 1; POLG, gamma DNA polymerase; SD, standard diet; SSBP1, single-strand DNA binding protein $1 ; \mathrm{STZ}$, streptozotocin.

Conflict of interest: The authors have declared that no conflict of interest exists. Citation for this article: J. Clin. Invest. 118:789-800 (2008). doi:10.1172/JCI32601. oxidize fatty acids, also play an important role in the development of insulin resistance (9). Indeed, the oxidative capacity in skeletal muscle, which is mostly dependent on mitochondrial function, is directly correlated with insulin sensitivity (10), and reduced mitochondrial oxidative phosphorylation is associated with insulin resistance (11). A reduction in the number and changes in the morphology of mitochondria has been observed in the skeletal muscle of type 2 diabetic patients (12). In addition, a set of genes involved in oxidative phosphorylation exhibits reduced expression levels in the muscle of type 2 diabetic patients and of prediabetic subjects $(13,14)$. These changes may be mediated by decreased expression of PPAR $\gamma$ coactivator $1 \alpha($ PGC1 $\alpha)$ and nuclear respiratory factor 1 (NRF1) genes, both of which control mitochondrial biogenesis. Interestingly, high-fat diets downregulated $P G C 1 \alpha$ and $P G C 1 \beta$ as well as genes coding for proteins of the electron transport chain in human skeletal muscle (15), which suggests that excess dietary fat could alter mitochondrial functions. However, the effects of excess dietary lipids on mitochondrial biogenesis and functions have not been investigated in detail, and the underlying mechanisms responsible for the reduced mitochondrial activity in the pathogenesis of insulin resistance are still unknown.

The purpose of this study was to determine the effects of a highfat, high-sucrose diet (HFHSD) on mitochondrial density and functions and on insulin action in mice skeletal muscle in order to (a) determine whether high fuel substrate availability contributes to mitochondrial dysfunction, (b) monitor the relationship between mitochondrial alterations and insulin resistance, and (c) identify the molecular mechanisms linking excess dietary fuels and altered mitochondrial functions. Our data show that HFHSD-induced mitochondrial alterations in skeletal muscle 


\section{Table 1}

\begin{tabular}{|c|c|c|c|c|c|c|c|}
\hline & \multicolumn{4}{|c|}{ SD/HFHSD protocol } & \multicolumn{3}{|c|}{ STZ/INS protocol } \\
\hline & SD 4 weeks & HFHSD 4 weeks & SD 16 weeks & HFHSD 16 weeks & Control & STZ & STZ+INS \\
\hline Body weight (g) & $24.08 \pm 0.38$ & $28.9 \pm 0.6^{A}$ & $27.7 \pm 0.36$ & $44 \pm 1.02^{\mathrm{A}}$ & $23.9 \pm 0.1$ & $19.6 \pm 0.1^{B}$ & $22 \pm 0.4 c$ \\
\hline Fat weight $(\mathrm{g})$ & $0.25 \pm 0.02$ & $1.1 \pm 0.07^{A}$ & $0.61 \pm 0.05$ & $2.5 \pm 0.08^{A}$ & ND & ND & ND \\
\hline Glucose (mg/dl) & $167 \pm 4.7$ & $170 \pm 4.7$ & $149 \pm 4.1$ & $210.7 \pm 7^{A}$ & $160 \pm 4$ & $598.5 \pm 21.6^{D}$ & $484.3 \pm 22^{\mathrm{E}}$ \\
\hline Insulin (ng/ml) & $0.87 \pm 0.2$ & $1.5 \pm 0.4^{F}$ & $0.8 \pm 0.22$ & $7.4 \pm 1.6^{A}$ & $0.8 \pm 0.2$ & $0.3 \pm 0.02^{D}$ & $15.4 \pm 3.3^{E}$ \\
\hline Leptin (pg/ml) & $3.1 \pm 0.3$ & $5.5 \pm 1.7^{\mathrm{F}}$ & $8.4 \pm 0.9$ & $55 \pm 3.7^{A}$ & ND & ND & ND \\
\hline Triglyceride (g/l) & $0.3 \pm 0.07$ & $0.2 \pm 0.03$ & $0.16 \pm 0.03$ & $0.4 \pm 0.06^{A}$ & ND & ND & ND \\
\hline FFA (mM) & $0.5 \pm 0.04$ & $0.6 \pm 0.13$ & $0.45 \pm 0.06$ & $0.9 \pm 0.1^{\mathrm{F}}$ & $0.11 \pm 0.01$ & $0.12 \pm 0.05$ & UD \\
\hline $\mathrm{H}_{2} \mathrm{O}_{2}(\mu \mathrm{M})$ & $29.9 \pm 8$ & $33.02 \pm 5.7$ & $28.8 \pm 3.4$ & $44 \pm 4.5^{\mathrm{F}}$ & ND & ND & ND \\
\hline
\end{tabular}

Data are expressed as mean \pm SEM of $10-20$ mice per group. ${ }^{A} P<0.001$ vs. respective $\mathrm{SD}$; ${ }^{B} P<0.05$ vs. control; ${ }^{C} P<0.05$ vs. $\mathrm{STZ}$; ${ }^{D} P<0.001$ vs. control; ${ }^{E} P<0.001$ vs. STZ; FP $<0.05$ vs. respective SD. ND, not determined; UD, undetected; STZ+INS, insulin-treated STZ.

are a consequence of hyperglycemia- and hyperlipidemia-induced ROS production in mice, which result from mitochondrial overfunctioning and an increase in $\mathrm{NAD}(\mathrm{P}) \mathrm{H}$ oxidase in response to energy substrate overflow.

\section{Results}

Metabolic characteristics of mice under HFHSD feeding. The metabolic characteristics of the mice are summarized in Table 1 . After 4 weeks of the diets, body weight was significantly greater $(20 \%$, $P<0.001)$ and epidydimal adipose tissue weight was significantly greater $(336 \%, P<0.001)$ in the HFHSD mice than in the mice fed standard diet (SD). Plasma glucose, FFA, and triglyceride levels were similar in both groups of mice, whereas plasma insulin $(72 \%, P<0.05)$ and leptin $(79 \%, P<0.05)$ levels were greater in the HFHSD mice than in the SD mice. After 16 weeks of the diets, body weight and epidydimal fat weight gains in the HFHSD mice were more marked than those in the SD mice, and the HFHSD mice were clearly hyperglycemic $(P<0.001)$ and hyperinsulinemic $(P<0.001)$ compared with the SD mice. In the HFHSD mice, plasma glucose levels were significantly greater at 16 weeks $(P<0.001)$ than at 4 weeks. At 16 weeks, plasma leptin $(P<0.001)$, FFA $(P<0.05)$, and triglyceride $(P<0.001)$ levels were all greater in the HFHSD mice than in the SD mice.

Glucose and insulin tolerance tests showed that HFHSD mice were glucose intolerant at 4 week, whereas their response to insulin injection remained unaltered compared with SD mice (Supplemental Figure 1, A and B; supplemental material available online with this article; doi:10.1172/JCI32601DS1). In contrast, after 16 weeks of feeding, the HFHSD mice presented an altered response to both glucose and insulin injection compared with the SD mice, which indicated that the HFHSD mice were insulin resistant (Supplemental Figure 1, A and B). Decreased insulin responsiveness in HFHSD mice at 16 weeks was associated with intramyocellular lipid accumulation (Supplemental Figure 2A), increased basal IRS1 serine phosphorylation at Ser632 (45\%, $P<0.05$; Supplemental Figure $2 \mathrm{~B}$ ), and a decrease in ex vivo insulin-stimulated Akt phosphorylation at Ser473 $(80 \%, P<0.01$; Supplemental Figure $2 \mathrm{C}$ ) in gastrocnemius muscle. In contrast, at 4 weeks, insulin-stimulated Ser473 phosphorylation of Akt was not significantly different between the HFHSD and SD mice (Supplemental Figure 2C).

Mitochondrial biogenesis is reduced in the skeletal muscle of HFHSD mice. Next, we investigated the impact of HFHSD on muscle mitochondrial density. As shown in Figure 1A, the ratio of mitochondrial
DNA (mtDNA) to nuclear DNA in the skeletal muscle was significantly lower in the HFHSD mice than in the SD mice $(3 \%, P<0.05)$ at 16 weeks, whereas no change was observed after 4 weeks of the diets. In agreement, the mRNA levels of the subunits 1 and 3 of cytochrome $c$ oxidase (COX), 2 mitochondria-encoded genes, were significantly lower in the skeletal muscle of the HFHSD mice than of the SD mice at 16 weeks (Figure 1B). Using transmission electron microscopy, we found that the amounts of both subsarcolemmal $(31 \%, P<0.05)$ and intermyofibrillar $(41 \%, P<0.01)$ mitochondria in oxidative fibers were lower in the HFHSD mice than in the SD mice at 16 weeks (Figure 1, C and D). These alterations were not observed after 4 weeks of HFHSD diet (Figure 1C). As shown in Figure 1E, citrate synthase (CS) activity was slightly lower in the mitochondria isolated from the HFHSD mice than in that from the SD mice after both 4 weeks $(13 \%, P<0.05)$ and 16 weeks $(16 \%$, $P<0.05)$ of feeding.

To clarify the mechanisms involved in the reduction of mitochondrial density in the muscle of the HFHSD mice at 16 weeks, we measured the mRNA levels of genes implicated in mitochondrial biogenesis, such as PGC1 $\alpha, P G C 1 \beta, N R F 1, N R F 2$, the mitochondrial transcription factor (mtTFA), estrogen-related receptor $\alpha(\mathrm{ERR} \alpha)$, and mitofusin 2 (Mfn2). Only the mRNA levels of PGC1 $\alpha$ and $M f n 2$ were lower in the skeletal muscle of the HFHSD mice than of the SD mice at 16 weeks ( $\sim 50 \%$ for both transcripts; Figure 2A). This difference was not seen after 4 weeks of the diets (Figure 2A). The protein levels of PGC1 $\alpha$ were also significantly decreased in the skeletal muscle of HFHSD mice compared with SD mice at 16 weeks (data not shown). Concerning mtDNA replication and repair, we investigated both gamma DNA polymerase (POLG1, the catalytic subunit, and POLG2, the accessory subunit) and the single-strand DNA binding protein 1 (SSBP1), which play a key role in this process (16). As illustrated in Figure 2B, 16 weeks of HFHSD feeding induced a decrease in POLG2 and SSBP1 mRNA levels in skeletal muscle, whereas no effect was observed after 4 weeks of feeding. POLG1 expression was not affected by HFHSD feeding.

Alteration of mitochondrial ultrastructure in the skeletal muscle of HFHSD mice. In addition to the observed reduction in mitochondrial content, the transmission electron microscopy study demonstrated marked alterations in mitochondrial morphology in the gastrocnemius muscle of the HFHSD mice at 16 weeks. Areas of both subsarcolemmal and intramyofibrillar mitochondria were lower $(45 \%$ and $35 \%$, respectively; $P<0.05)$ in the skeletal muscle 
A
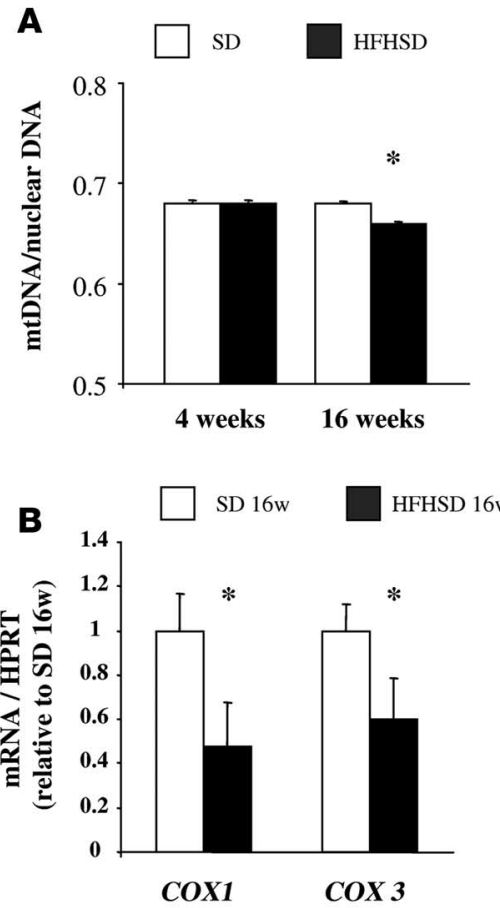

C

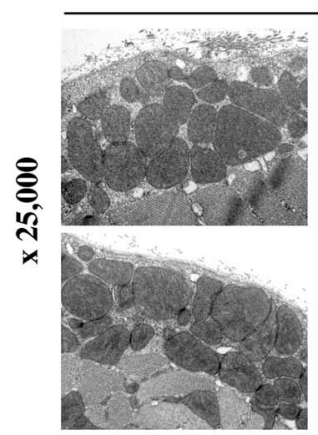

SD

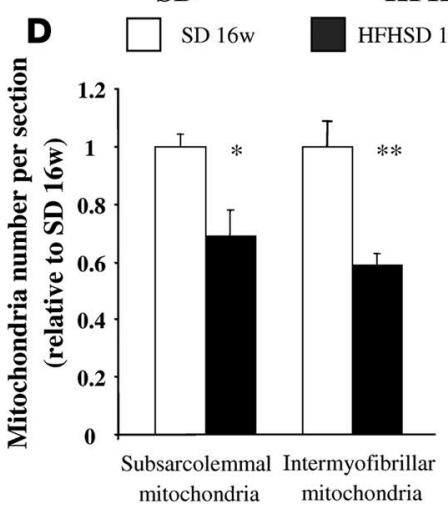

\section{Intramyofibrillar mitochondria}

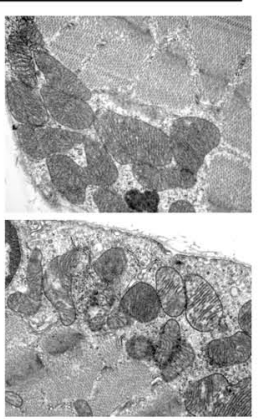

HFHSD

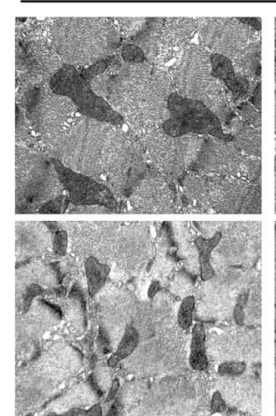

SD

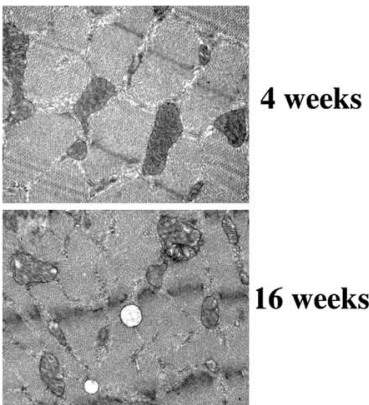

HFHSD

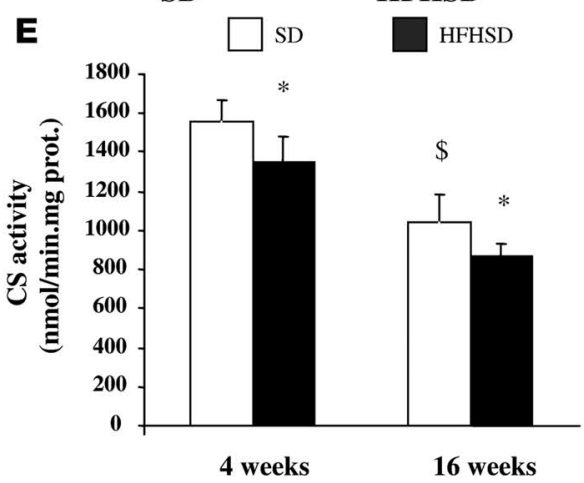

Figure 1

Decreased mitochondrial density in the skeletal muscle of mice fed the HFHSD for 16 weeks. (A) mtDNA quantity calculated as the ratio of COX1 to cyclophilin A DNA levels determined by real-time PCR in the skeletal muscle of mice after 4 and 16 weeks of the SD and HFHSD $(n=6)$. Note that the scale of the $y$ axis is between 0.5 and 0.8 . (B) mRNA expression of mitochondria-encoded COX1 and COX3 genes determined by quantitative RT-PCR in the skeletal muscle of mice fed the HFHSD for 16 weeks $(n=6)$. Results were normalized by the mean value for the SD mice at 16 weeks set to 1 unit. (C) Mitochondrial density assessed by electron microscopy in the skeletal muscle of mice after 4 and 16 weeks of the SD or HFHSD. Original magnification, $\times 25,000$. (D) Quantification of subsarcolemmal and intermyofibrillar mitochondria number per image area in the gastrocnemius muscle of mice after 16 weeks of the HFHSD (analysis of 5 images in 3 mice per group). Results were normalized by the mean value for the SD mice at 16 weeks set to 1 unit. (E) CS activity in mitochondria isolated from the gastrocnemius muscle after 4 and 16 weeks of the SD or HFHSD $(n=6) .{ }^{*} P<0.05,{ }^{* *} P<0.01$ vs. SD; ${ }^{\$} P<0.05$ vs. SD at 4 weeks.

of the HFHSD mice than of the SD mice at 16 weeks (Figure 3, A and C). Higher magnification $(\times 100,000)$ showed swelling of both types of mitochondria associated with an increased number of disarrayed cristae and a reduced electron density of the matrix (Figure 3B). No alterations in mitochondrial morphology were observed after 4 weeks of the HFHSD (data not shown).

Altered mitochondrial function in the skeletal muscle of HFHSD mice. To investigate whether alterations in mitochondrial density and ultrastructure were associated with mitochondrial dysfunction in the skeletal muscle of HFHSD mice, we measured substratedriven oxygen consumption in saponin-skinned skeletal muscle fibers. The respiration rates of mice fed an HFHSD for 4 weeks were not significantly different from those of the SD mice, regardless of the tested substrates (Table 2). Compared with the SD mice, respiration in muscle fibers with complex 1 -linked substrates (glutamate/malate), but not with complex 2-linked substrates (succinate/rotenone), was significantly reduced in HFHSD mice at 16 weeks, both during state 3 and state 4 (Table 2). In addition, we observed a significant decrease in oxidation capacities at 16 weeks when using octanoyl- or palmitoyl-carnitine as substrates in fibers of HFHSD mice. Taken together, these data demonstrate that complex 1 -linked respiration and $\beta$-oxidation were decreased specifically in diet-induced diabetic mice. Reduced oxidation of fatty acids was probably not related to altered availability of the substrates because genes involved in muscle fatty acid uptake (FAT/CD36) and entry in the mitochondria (CPT1) were significantly upregulated in the skeletal muscle of HFHSD mice at 16 weeks (Supplemental Figure 3A). In further support of a reduction in mitochondrial functions, a decrease in the activity of succinate dehydrogenase was evidenced by succinate dehydrogenase staining in histological sections of gastrocnemius muscle from HFHSD mice at 16 weeks but not at 4 weeks (Supplemental Figure 3B).

Increased oxidative stress in the skeletal muscle of HFHSD mice. Because mitochondrial alterations were observed in HFHSD mice only when they were hyperglycemic and hyperlipidemic, and because both glucose and lipids are known to induce oxidative stress, we tested whether ROS levels were increased during HFHSD feeding. Plasma $\mathrm{H}_{2} \mathrm{O}_{2}$ levels (Table 1 ) and muscular protein carbonylation levels (a marker of protein oxidation; Figure 4A) were elevated in HFHSD mice compared with SD mice at 16 weeks. No differences were observed after 4 weeks of the diets (Figure 4A).

In addition, mRNA levels of uncoupling proteins 2 and 3 (markers of increased mitochondrial ROS production in conditions of lipid oversupply; ref. 17) and of 4 subunits of $\mathrm{NAD}(\mathrm{P}) \mathrm{H}$ oxidase 
A

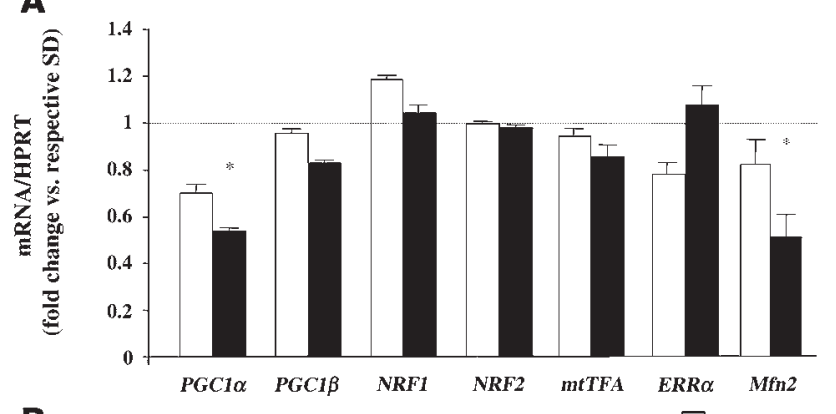

B

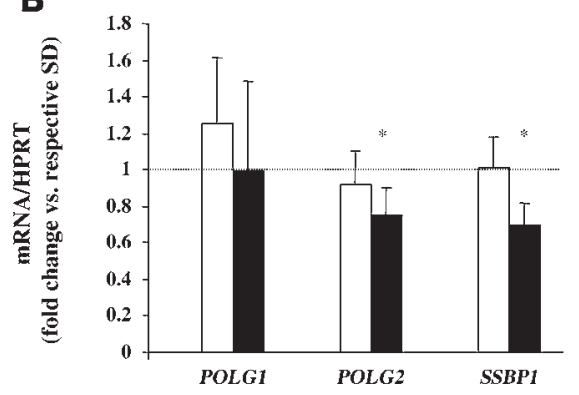

Figure 2

Expression of genes implicated in mitochondrial biogenesis and in mtDNA replication. mRNA levels of mitochondrial biogenesis $(\mathbf{A})$ and mtDNA replication (B) genes, determined by quantitative RT-PCR, in the gastrocnemius muscle of the mice after 4 and 16 weeks of the SD or HFHSD $(n=6)$. Results are expressed as fold change versus the $\mathrm{SD}$ diet set to 1 unit (dotted line). ${ }^{*} P<0.05$.

$(g p 91, p 67, p 40$, and $p 47)$ were induced in the skeletal muscle of HFHSD mice at 16 weeks, which suggests an increase in both mitochondrial and cytoplasmic ROS production (Figure 4B). Only the p67 subunit of $\mathrm{NAD}(\mathrm{P}) \mathrm{H}$ oxidase was significantly induced after 4 weeks of HFHSD feeding. Concerning the antioxidant system, the mRNA levels of glutathione reductase and catalase were increased in the skeletal muscle of HFHSD mice at 16 weeks, whereas expression of other antioxidant enzymes, including glutathione peroxidase, superoxide dismutase 2, peroxiredoxin 3, and peroxiredoxin 5 , were not modified (Figure 4B). None of these genes showed a modification of expression after 4 weeks of the HFHSD.

Exposure to ROS leads to apoptosis and cell damage in a variety of experimental systems. As shown in Figure 4C, we observed an increase in cytochrome $c$ levels in the cytosol and a concomitant decrease in the mitochondria fraction of skeletal muscle in HFHSD mice at 4 weeks, which indicated the release of cytochrome $c$ from mitochondria. Because of the strong alterations in the number and functions of mitochondria in the muscle of HFHSD-fed mice at 16 weeks, this phenomenon was difficult to observe after 16 weeks of feeding (Figure 4C). Nevertheless, the activity of caspase 3, another index of apoptosis, was markedly increased in the skeletal muscle of HFHSD mice at 16 weeks $(90 \%, P<0.05)$, whereas no difference was observed after 4 weeks of the diet (Figure 4D).

We also investigated ROS production and mitochondrial dysfunction in $\mathrm{KKA}^{y}$ mice, a genetic model of obesity and diabetes. $\mathrm{KKA}^{\mathrm{y}}$ mice were obese, hyperglycemic, hyperinsulinemic, and hypertrygliceridemic compared with age-matched control mice (Supplemental Table 1), but they had normal plasma FFA levels (Supplemental Table 1). Plasma $\mathrm{H}_{2} \mathrm{O}_{2}$ (Supplemental Table 1), but not skeletal muscle protein carbonylation (Supplemental Figure $4 \mathrm{~A})$, was greater in $\mathrm{KKA}^{\mathrm{y}}$ mice than in $\mathrm{C} 57 \mathrm{BL} / 6$ mice. Interestingly, in contrast with the HFHSD model, mitochondrial density and structure were not altered in KKAy mice compared with agematched control mice (Supplemental Figure 4, B and C). Taken together, these data suggest that oxidative stress in skeletal muscle is a determinant of mitochondrial alterations in diabetic mice.

Alteration of mitochondria biogenesis and structure in the skeletal muscle of streptozotocin-treated mice. To test whether ROS production is a key feature in HFHSD-induced mitochondrial dysfunction, we investigated mitochondrial structure and function in mice treated with streptozotocin (STZ), a model of hyperglycemia-associated oxidative stress with no insulin resistance and obesity. Eleven days after STZ administration, the mice were hyperglycemic $(P<0.001)$ and hypoinsulinemic $(P<0.001)$, had no changes in plasma FFA levels, and had a reduction in body weight $(P<0.005$; Table 1$)$. Insulin injection of the STZ mice rapidly decreased plasma glucose levels; 24 hours after insulin injection, plasma glucose levels were lower $(P<0.001)$, body weights were higher $(P<0.05)$, and FFA levels were undetectable in insulin-injected STZ mice compared with STZ mice (Table 1). Phlorizin injection of STZ mice reproduced the effect of insulin on glycemia: glucose decreased by $25 \%$ compared with STZ mice $(P<0.05)$.

In agreement with the observed hyperglycemia-induced oxidative stress, protein carbonylation levels were elevated in the skeletal muscle of STZ mice, and insulin treatment restored the extent of protein carbonylation to levels close to those observed in control mice (Figure 5A). Furthermore, STZ treatment induced a release of cytochrome $c$ from mitochondria, and insulin treatment reversed this proapoptotic process (Figure 5B). Regarding mitochondrial density, the mtDNA/nuclear DNA ratio (Figure 5C) and amount of mitochondria per area (Figure 5D) were reduced in the muscle of STZ mice compared with control mice. The morphology of both types of mitochondria was also affected in the skeletal muscle of STZ mice; the number of cristae was reduced, and the electron density of the matrix decreased (Figure 5D). Importantly, density and structural abnormalities of mitochondria in the muscle of STZ mice were restored by insulin and phlorizin treatments (Figure 5, C and D).

To verify whether mitochondrial alterations were related to ROS production, we treated STZ mice with $\mathrm{N}$-acetylcysteine (NAC), a general antioxidant. NAC treatment did not modify systemic oxidative stress (Figure 6A), but did decrease muscle protein carbonylation to the levels of control mice (Figure $6 \mathrm{~B}$ ) and restored mitochondria density (Figure 6C) and structure (Figure 6D) in the gastrocnemius muscle of STZ mice.

Taken together, these results demonstrate that oxidative stress in hyperglycemic mice is associated with altered mitochondrial structure and function in skeletal muscle and that both the amelioration of glycemia and antioxidant treatment restored mitochondrial structure.

ROS induce mitochondrial alterations and dysfunction in cultured myotubes. We examined the effects of high glucose and lipid levels on ROS production and mitochondria density and functions in $\mathrm{C}_{2} \mathrm{C}_{12}$ muscle cells. ROS production was markedly increased by glucose $(25 \mathrm{mM})$ and by palmitate $(200 \mu \mathrm{M})$ treatments for 96 hours, and the addition of NAC (10 mM) blocked these effects (Figure 7A). The addition of $\mathrm{H}_{2} \mathrm{O}_{2}(100 \mu \mathrm{M})$ for 96 hours decreased mtDNA levels (Figure 7B) and reduced CS activity (Figure 7C) in $\mathrm{C}_{2} \mathrm{C}_{12}$ cells. Incubation with glucose or with palmitate also decreased CS 
A

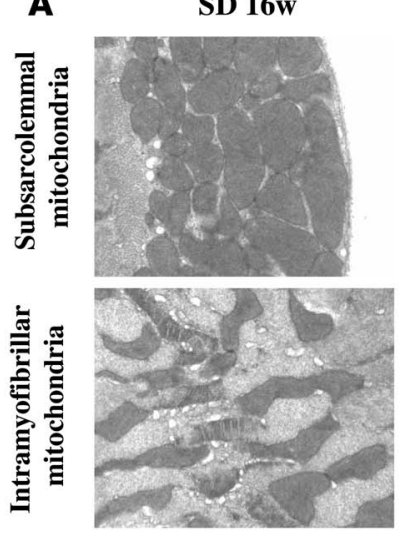

B
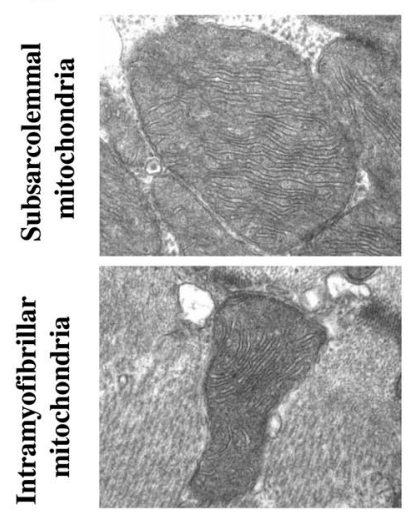

HFHSD 16w

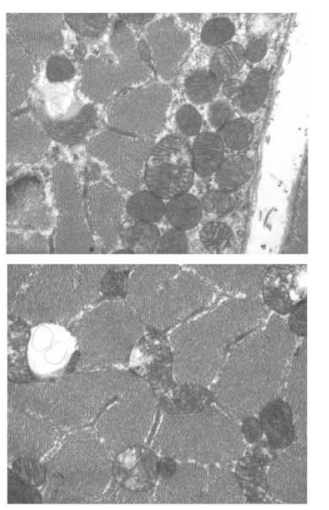

HFHSD 16w

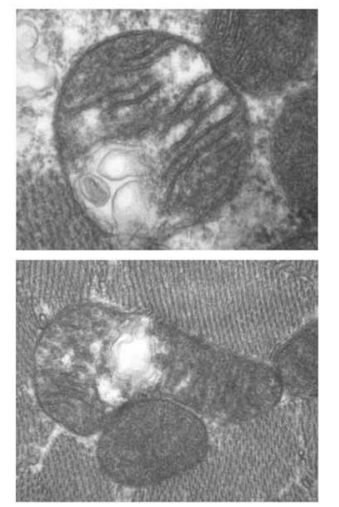

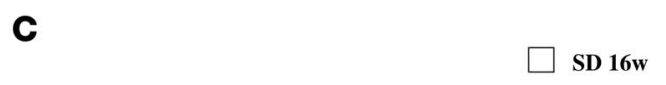

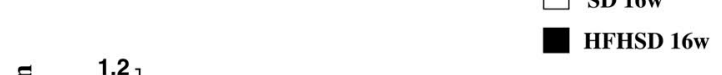

हू
ปิ
$x$

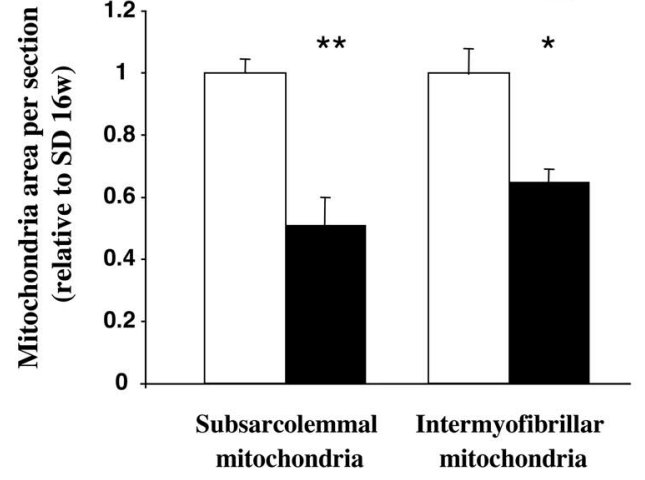

Figure 3

Alterations in the mitochondrial structure of skeletal muscle of mice fed the HFHSD for 16 weeks. (A and B) Transmission electron microscopy images at original magnifications of $\times 25,000(\mathbf{A})$ and $\times 100,000$ (B) in subsarcolemmal and intermyofibrillar mitochondria from the gastrocnemius muscle of mice after 16 weeks of the SD or HFHSD. (C) Quantification of subsarcolemmal and intermyofibrillar mitochondria area in the gastrocnemius muscle of mice fed the HFHSD for 16 weeks (analysis of 5 images in 3 mice per group). Results were normalized by the mean value for the SD mice at 16 weeks set to 1 unit. ${ }^{*} P<0.05,{ }^{* *} P<0.01$ vs. SD.

activity (Figure 7C), but the effects on mtDNA were not significant (Figure 7B). Furthermore, POLG2, SSBP1, and PGC1 $\alpha$ mRNA levels were decreased in myotubes treated with $\mathrm{H}_{2} \mathrm{O}_{2}$, glucose, or palmitate for 96 hours. The addition of NAC counteracted all these effects, which indicated that ROS contributed to the observed mitochondrial alterations in cultured muscle cells (Figure 7, B-D). Finally, we also performed experiments in primary cultures of human myotubes and found similar results (Supplemental Figure 5), which suggests that these effects could also take place in human muscle cells. Transmission electron microscopy studies have nicely illustrated that the addition of both $\mathrm{H}_{2} \mathrm{O}_{2}$ and glucose for 96 hours altered mitochondria structure in myotubes compared with their respective control cells (Supplemental Figure 5D).

\section{Discussion}

Cumulative evidence strongly suggests that alterations in mitochondrial density and function in skeletal muscle are associated with both insulin resistance and type 2 diabetes (9). However, whether these changes are a cause, a consequence, or a parallel process of insulin resistance is not clear. Here, we investigated the amount, structure, and function of skeletal muscle mitochondria during the development of HFHSD-induced insulin resistance in mice. Our data indicate that mitochondrial defects do not appear before insulin resistance because no changes were observed in a prediabetic state (after 4 weeks of diet), whereas mitochondrial dysfunction was evident in the skeletal muscle of diabetic mice (after 16 weeks of diet). Furthermore, we found that oxidative stress in skeletal muscle is probably one of the major determinants of mitochondrial alterations. This is supported by data showing that (a) an increase in muscle ROS production occurred specifically after 16 weeks of the HFHSD when the mice were hyperglycemic and hyperlipidemic; (b) ROS production was also associated with mitochondrial alterations in the muscle of hyperglycemic STZ mice; (c) in this model, normalization of glycemia by insulin or phlorizin and treatment with the antioxidant NAC decreased muscle ROS production and restored mitochondrial integrity; (d) incubation of cultured muscle cells with high glucose or high lipid concentrations induced ROS production and altered mitochondrial density and functions; and (e) these effects were blocked by antioxidant treatment.

We investigated mitochondrial structure and functions in the skeletal muscle of mice at 2 different stages of HFHSD-induced metabolic disturbances. After 4 weeks, mice were overweight, normoglycemic, and normolipidemic and had no systemic or muscle oxidative stress. However, they showed hyperinsulinemia and altered glucose clearance during a glucose tolerance test, but 


\section{Table 2}

Respiration rates and respiration control ratio in permeabilized muscle fibers of mice after 4 and 16 weeks of the SD and HFHSD

\begin{tabular}{lccccc} 
& & \multicolumn{4}{c}{ Respiration rate [nat 0/(min.mg dry weight)] } \\
& & SD & HFHSD & SD & HFHSD \\
Glutamate/malate & & $\mathbf{4}$ weeks & 4 weeks & $\mathbf{1 6}$ weeks & 16 weeks \\
& State 4 & $2.45 \pm 0.3$ & $2.55 \pm 0.2$ & $2.23 \pm 0.1$ & $1.62 \pm 0.2^{\mathrm{A}}$ \\
& State 3 & $6.43 \pm 0.9$ & $6.15 \pm 0.8$ & $6.34 \pm 0.4$ & $4.5 \pm 0.4^{\mathrm{A}}$ \\
Succinate/rotenone & RCR & $2.79 \pm 0.5$ & $2.47 \pm 0.3$ & $2.86 \pm 0.2$ & $2.87 \pm 0.3$ \\
& State 4 & $5.02 \pm 0.6$ & $6.61 \pm 0.9$ & $5.25 \pm 0.5$ & $5.55 \pm 0.7$ \\
& State 3 & $10.7 \pm 1.4$ & $14.4 \pm 1.2$ & $11.85 \pm 1.4$ & $11.1 \pm 0.9$ \\
Octanoyl-carnitine/malate & RCR & $2.79 \pm 0.5$ & $2.47 \pm 0.4$ & $2.86 \pm 0.2$ & $2.87 \pm 0.3$ \\
& State 4 & $1.86 \pm 0.1$ & $1.36 \pm 0.1$ & $1.4 \pm 0.1$ & $0.99 \pm 0.1$ \\
& State 3 & $3.93 \pm 0.4$ & $3.52 \pm 0.5$ & $3.38 \pm 0.5$ & $1.73 \pm 0.2^{\mathrm{A}}$ \\
Palmitoyl-carnitine/malate & RCR & $2.11 \pm 0.18$ & $2.66 \pm 0.5$ & $2.29 \pm 0.4$ & $1.85 \pm 0.2$ \\
& State 4 & $1.18 \pm 0.2$ & $1.49 \pm 0.1$ & $1.47 \pm 0.2$ & $1.01 \pm 0.1$ \\
& State 3 & $2.19 \pm 0.3$ & $2.99 \pm 0.3$ & $3.68 \pm 0.6$ & $1.72 \pm 0.26^{\mathrm{A}}$ \\
& RCR & $1.95 \pm 0.2$ & $2.07 \pm 0.3$ & $2.65 \pm 0.4$ & $2.08 \pm 0.3$
\end{tabular}

Respiration rates were measured on saponin-skinned fibers as described in Methods. State 4 was the control state of respiration in presence of actractyloside, and state 3 was the ADP-stimulated respiration. Respiration control ratio (RCR) was calculated as state3/state 4. Values are mean \pm SEM for 6 animals per group. ${ }^{A} P<0.01$ vs. respective SD. nat, nanoatoms.

lipid accumulation and insulin resistance. Nevertheless, these studies were conducted in subjects who were already insulin resistant $(11,19)$; thus, it was not possible to determine whether mitochondrial alterations precede the development of insulin resistance.

HFHSD feeding for 16 weeks was associated with hyperglycemia, hyperinsulinemia, increased plasma and muscle lipid levels, and altered in vivo and ex vivo insulin responsiveness, which indicated that the HFHSD mice were diabetic at 16 weeks. These metabolic alterations were associated with systemic and muscle oxidative stress, probably because of an increase in both mitochondria and cytoplasmic ROS production rather than to a reduction in antioxidant defenses. Furthermore, these disturbances were associated with striking mitochondrial changes in gastrocnemius muscle. There was a significant decrease in mitochondria number associated with a reduction in mtDNA content and reduced expres-

normal in vivo and ex vivo insulin responsiveness. Consequently, HFHSD-fed mice could have been considered to be in a prediabetic state at 4 weeks because they were glucose intolerant but did not have diabetes. At this time, we did not observe any changes in mitochondrial density and structure in skeletal muscle, as assessed by electron microscopy. mtDNA copy number, as well as the expression of mitochondria-encoded genes and key regulators of mitochondrial biogenesis, was not altered in the skeletal muscle of these prediabetic mice. Furthermore, mitochondrial functions in the muscle of HFHSD mice at 4 weeks were normal, as assessed by substrate-driven respiration and lipid oxidative capacities measurements. Taken together, these data clearly indicate that mitochondrial alterations do not precede the onset of insulin resistance and diabetes. The only early perturbations observed in the skeletal muscle of HFHSD mice at 4 weeks were as follows: (a) a slight increase in lipid stores (data not shown), which indicates a preferential orientation of muscle metabolism toward lipid esterification in the prediabetic state, as previously reported in humans (18); (b) a release of cytochrome $c$ from mitochondria, which suggests that early proapoptotic events in skeletal muscle could precede mitochondrial alterations during an HFHSD; (c) a decrease in CS activity that could be attributed to the HFHSDinduced downregulation of CS gene expression, as supported by reduced CS mRNA levels in the muscle of the HFHSD mice compared with the SD mice at 4 weeks (CS/hypoxanthine guanine phosphoribosyl transferase [CS/HPRT] mRNA, $0.29 \pm 0.03$ vs. $0.45 \pm 0.03 ; P<0.01$ ). Although these results suggest that 4 weeks of HFHSD feeding was associated with some metabolic perturbations, which may reflect the initiation of deleterious processes, there was no major functional or structural alteration in the mitochondria in the skeletal muscle of the prediabetic mice. Recent studies in humans, however, indicated impaired mitochondrial activity and density in the skeletal muscle of the offspring of type 2 diabetic patients $(11,19)$, which leads to the concept that early mitochondrial alterations could predispose to intramyocellular sion levels of mitochondria-encoded genes (COX1 and COX3), which suggested that the control of mitochondrial biogenesis and/or mtDNA replication is altered in diabetic mice. PGC1 $\alpha$ is one of the master regulators of mitochondrial biogenesis and oxidative phosphorylation gene expression (20). Two DNA microarray studies have found a coordinated reduction in the expression of genes regulated by PGC1 $\alpha$ in the skeletal muscle of type 2 diabetic patients $(13,14)$, and the expression of PGC1 $\alpha$ itself is decreased in the muscle of the patients $(14,21)$. In agreement, we found that $P G C 1 \alpha$ was downregulated in the skeletal muscle of HFHSD mice at 16 weeks, but we did not observe significant changes in downstream targets of PGC1 $\alpha$, such as NRF1, NRF2, $m t T F A$, and $E R R \alpha$. We observed, however, decreased expression of $M f n 2$, a protein participating in the mitochondrial network. This observation agrees with previous reports in other models of obesity and diabetes and also in humans (22). We also reported, for the first time to our knowledge, a decrease in POLG2 and SSBP1 expression in the skeletal muscle of diabetic mice, which was reproduced in vitro by $\mathrm{H}_{2} \mathrm{O}_{2}$, glucose, and lipid treatments and restored by NAC treatment. These results suggest that the altered expression of POLG2 and SSBP1 was probably a consequence of increased ROS production. Together with a decreased mtDNA content, these data indicate alterations in mtDNA replication in the skeletal muscle of diabetic mice. HFHSD feeding was also associated with decreased mitochondrial functions because substrate-driven oxygen consumption was altered, specifically in the muscle fibers of HFHSD mice at 16 weeks. State 3 and state 4 respiration rates were reduced when glutamate/malate were used as substrates, which indicated a decrease in the oxidation of $\mathrm{NADH}_{2}$ at complex 1. Furthermore, decreased oxygen consumption, with octanoyl- and palmitoyl-carnitine as substrates, suggested an impaired $\beta$-oxidation rate in the muscle of HFHSD mice at 16 weeks. These defects in respiratory functions could be secondary to decreases in mitochondria content. However, the fact that respiration with succinate/rotenone was not altered, indicating 
A

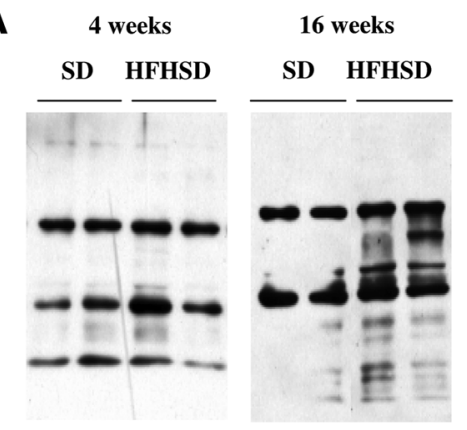

B

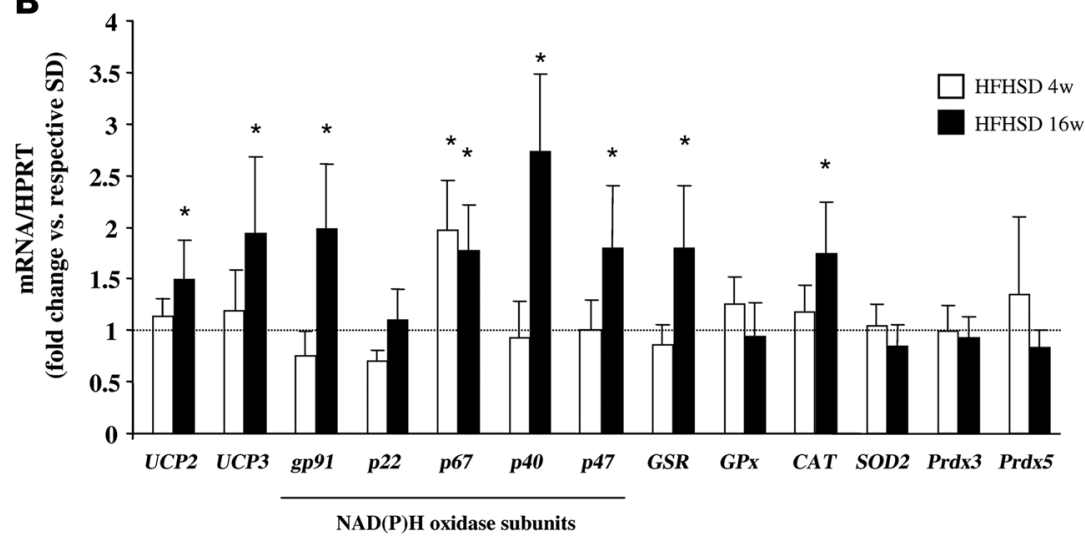

C

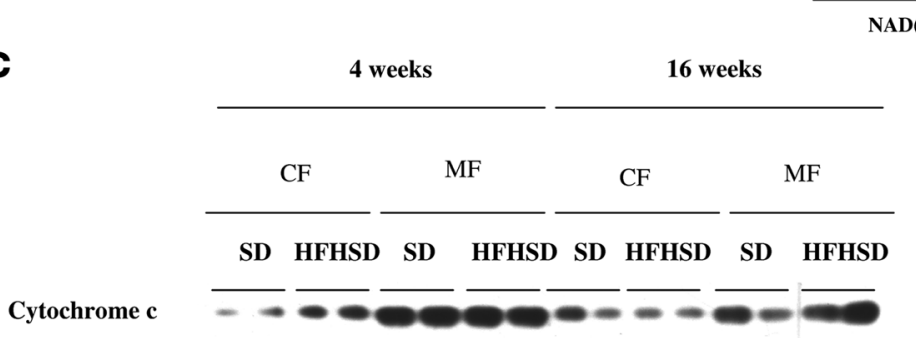

$11 \mathrm{kDa}$

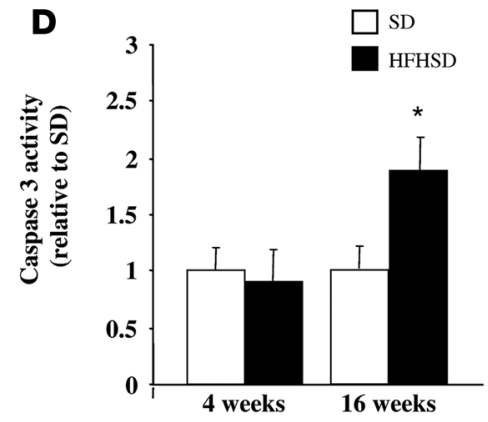

Figure 4

Chronic HFHSD feeding induces oxidative stress in skeletal muscle. (A) Immunoblots showing total protein carbonylation in the gastrocnemius muscle of mice after 4 and 16 weeks of the SD and HFHSD. (B) mRNA levels of oxidant stress-related genes determined by real-time RT-PCR in the gastrocnemius muscle of mice after 4 and 16 weeks of the SD and HFHSD $(n=6)$. Results are expressed as fold change versus the SD diet set to 1 unit (dotted line). ${ }^{*} P<0.05$. (C) Immunoblot showing cytochrome $c$ protein in the mitochondrial fraction (MF) and cytosolic fraction (CF) of the gastrocnemius muscle of mice after 4 and 16 weeks of the SD and HFHSD. (D) Caspase 3 activity measured in the gastrocnemius muscle of mice after 4 and 16 weeks of the HFHSD $(n=6)$. Results were normalized by the mean value for the SD mice at 4 and 16 weeks. ${ }^{*} P<0.05$. UCP, uncoupling protein; GSR, glutathione reductase; GPx, glutathione peroxidase; CAT, catalase; SOD, superoxide dismutase; Prdx, peroxiredoxin.

normal oxidation rates of $\mathrm{FADH}_{2}$ at complex 2, strongly suggests that specific alterations in mitochondria functions occurred in the muscle of HFHSD mice at 16 weeks.

A striking phenotype of skeletal muscle in diabetic mice resided in the structural anomalies of the mitochondria, as revealed by electron microscopy. Both subsarcolemmal and intramyofibrillar mitochondria were affected, which indicated common alterations independent of the subcellular localization. A number of mitochondria appeared swollen, with fewer cristae, and the inner and/or outer membranes were sometimes disrupted in the muscle of HFHSD mice at 16 weeks. The same mitochondrial alterations, including decreased mitochondrial density, mitochondrial swelling and disruption, and reduced mtDNA copy number, were observed in the skeletal muscle of STZtreated mice - a well-known model associated with hyperglycemia-induced oxidative stress. STZ mice were hyperglycemic and hypoinsulinemic, but they were not insulin resistant. In agreement, administration of exogenous insulin improved circulating concentrations of glucose, restored glycogen and lipid stores in muscle, and decreased oxidative injury, as evidenced by a reduction in protein carbonylation. In parallel, mitochondrial density, structural alterations, and mtDNA copy number improved in the skeletal muscle of insulin-treated STZ mice. The finding that phlorizin treatment decreased glycemia and was associated with an increase in the mtDNA content and an improvement in mitochondrial density and structure in skeletal muscle confirms the finding that mitochondrial restoration was secondary to the improvement in glycemia.

To confirm the implication of oxidative stress in skeletal muscle mitochondrial alterations, we demonstrated that antioxidant treatment of STZ mice restored mitochondrial density and structure. The strong analogies between HFHSD and STZ regarding the changes in mitochondria structure and integrity in skeletal muscle strongly suggest common underlying mechanisms. Oxidative stress in skeletal muscle, induced by hyperglycemia in STZ mice and the combination of hyperglycemia and hyperlipidemia in HFHSD mice, could be the culprit. In support of this assumption, in vitro data in cultured skeletal muscle cells demonstrated that treatment with high glucose or high fatty acid concentrations induced ROS production and mitochondrial damage in myotubes. This assumption is also consistent with several reports that showed that high glucose levels (23) as well as elevated fatty acids (24) increase oxidative stress in various models. Interestingly, the absence of ROS production in the muscle of $\mathrm{KKA}^{\mathrm{y}}$ mice further suggests that weak hyperglycemia, in the absence of elevated FFA levels, is not sufficient to increase ROS production and mitochondrial dysfunction. We were unable to determine whether ROS production is the only factor contributing to mitochondrial dysfunction. However, the fact that the addition of $\mathrm{H}_{2} \mathrm{O}_{2}$ induced a decrease in the amount of mtDNA and CS activity in cultured myotubes, and a concomitant 
A

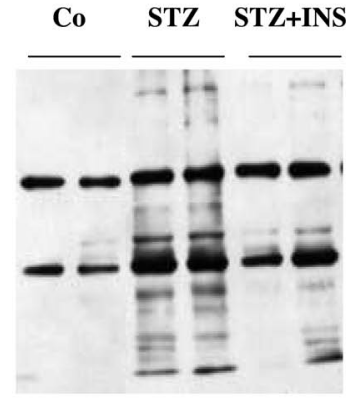

B

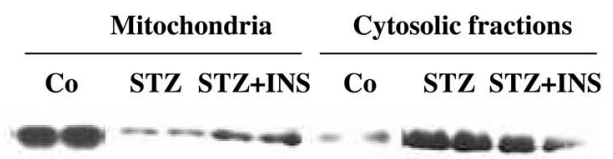

Cyt.

C

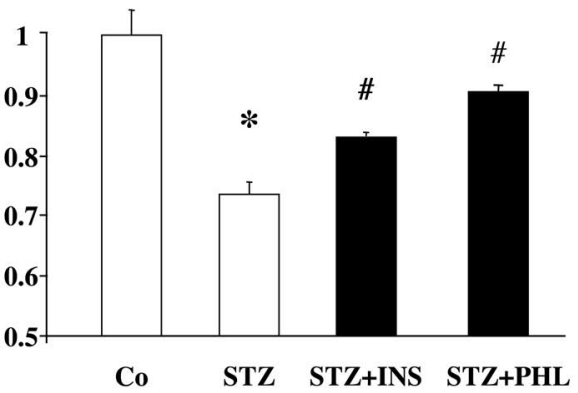

D

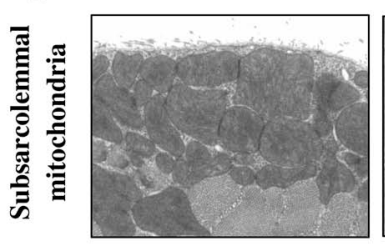

흘

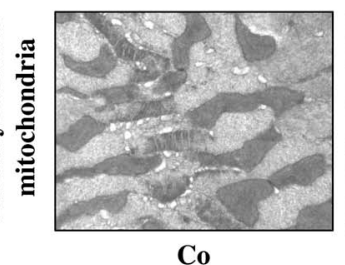

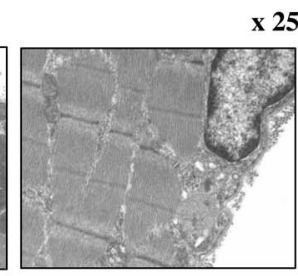

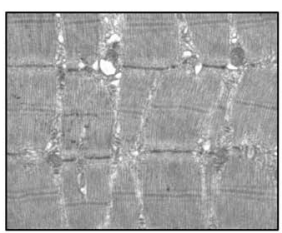

STZ x 25,000

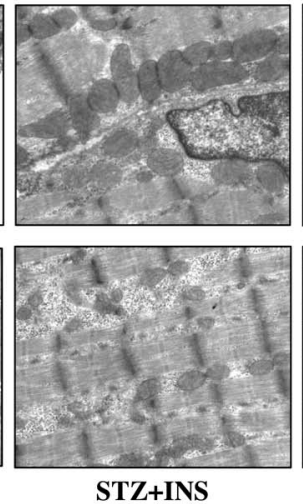

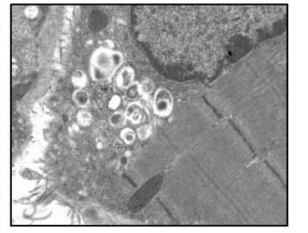

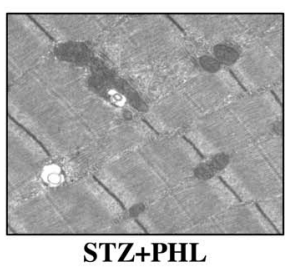

\section{Figure 5}

STZ-induced oxidative stress alters mitochondria density and structure in skeletal muscle. (A) Immunoblots showing total protein carbonylation in the gastrocnemius muscle of control (Co), STZ, and insulin-treated STZ (STZ+INS) mice. (B) Immunoblot showing cytochrome $C$ protein in the $\mathrm{MF}$ and CF of the gastrocnemius muscle of control, STZ, and insulin-treated STZ mice. (C) mtDNA copy number was calculated as the ratio of COX1 to cyclophilin A DNA levels, determined by real-time PCR, in the skeletal muscle of control, STZ, insulin-treatd STZ, and phlorizin-treated STZ (STZ+PHL) mice $(n=6)$. Note that the $y$ axis scale is between 0.5 and 1 . Results were normalized by the mean value for the control mice set to 1 unit. ${ }^{*} P<0.01$ vs. control; " $P<0.05$ vs. STZ. (D) Transmission electron microscopy images (original magnification, $\times 25,000$ ) of subsarcolemmal and intermyofibrillar mitochondria from the gastrocnemius muscle of control, STZ, insulin-treated STZ, and phlorizin-treated STZ mice. reduction in POLG2 and SSBP1 expression, and that these effects were reversed by antioxidant treatment supports a critical role of ROS in mediating mitochondria alterations in skeletal muscle. In agreement with this conclusion, it has been shown that glucoseinduced ROS production and oxidative stress in dorsal root ganglion neurons paralleled changes in mitochondrial size and function (25). In addition, mitochondrial DNA polymerase has been shown to be one of the targets of oxidative damage (26).

What is the mechanism of ROS-induced mitochondrial dysfunction? It seems that increased ROS production in skeletal muscle is crucial for the induction of mitochondrial alterations because mitochondrial density and structure were not altered in genetically obese and diabetic KKAy mice, which were hyperglycemic and had mildly elevated plasma $\mathrm{H}_{2} \mathrm{O}_{2}$ levels but no intramuscular oxidative stress, as evidenced by low levels of muscle protein carbonylation. In addition, restoration of mitochondria damage in NAC-treated STZ mice was associated with a decrease in the index of skeletal muscle oxidative stress, but not in plasma $\mathrm{H}_{2} \mathrm{O}_{2}$ levels. These findings also suggest that local oxidative stress might be determinant for mitochondria alterations in skeletal muscle. Moreover, investigations of the changes in the expression of several enzymatic systems involved in the regulation of oxidative stress in muscle revealed increases in the mRNA levels of uncoupling proteins and of almost all the subunits of $\mathrm{NAD}(\mathrm{P}) \mathrm{H}$ oxidase, which strongly suggests that locally increased ROS production was probably due to de novo mitochondrial and cytoplasmic generation, rather than to reduced antioxidant defenses during HFHSD. We propose the following working hypothesis to explain how skeletal muscle oxidative stress could induce mitochondrial dysfunction during HFHSD in mice: chronic elevation of plasma glucose and fatty acid levels leads to an overflow of energy substrates in muscle, which promotes intramyocellular lipid accumulation and inducing ROS production through increased mitochondrial uncoupling (17) and increased $\mathrm{NAD}(\mathrm{P}) \mathrm{H}$ oxidase enzyme (23). This intramuscular oxidative stress then causes mitochondria alterations and decreases mitochondria functions through damages of proteins, lipids, and DNA (27). Particularly, an increase in the ROS level could lead to the decreased expression of PGC1 $1, P O L G 2$, and SSBP1 and to altered mitochondrial biogenesis and mtDNA replication, which in turn contributes to mitochondrial dysfunction. Consequently, fatty acid oxidation is dampened, which amplifies the deposition of lipids in muscle. This initiates a vicious cycle in which increased intramuscular lipids, prone to ROS-induced formation of lipid peroxides (28), could foster mitochondrial damage. Another potential mechanism, which needs further investigation, could also implicate a ROS-mediated regulation of sirtuin activity. Indeed, it was recently demonstrated that muscle mitochondrial function is controlled by the activation of both deacetylase sirtuin 1 and PGC1 $\alpha$ $(29,30)$. Moreover, resveratrol treatment, which likely increases sirtuin activity, decreases PGC1 $\alpha$ acetylation, improves mitochondrial function, and protects mice against diet-induced obesity and diabetes (30). Because resveratrol has antioxidant capacities, it is tempting to speculate that ROS-induced mitochondrial dysfunction could involve decreased sirtuin activity and increased acetylation of proteins, including PGC1 $\alpha$. In agreement, acetylation of PGC1 $\alpha$ was shown to be elevated in mice fed a high-fat diet (30). 
A

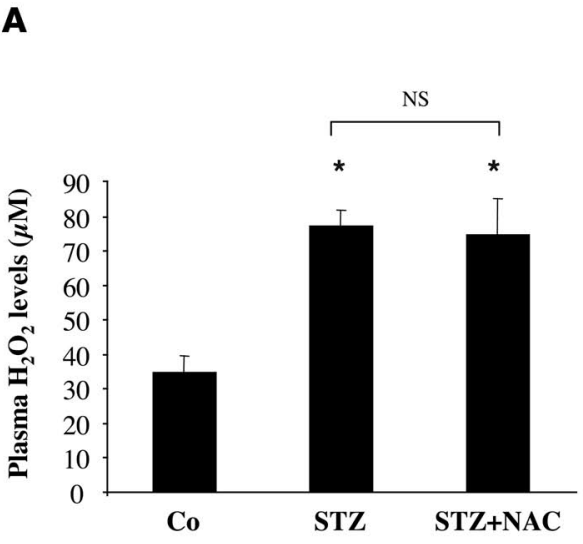

B

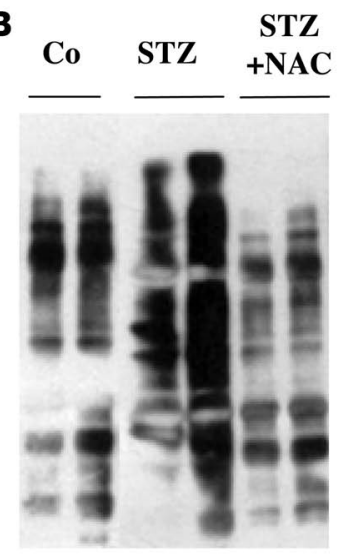

C

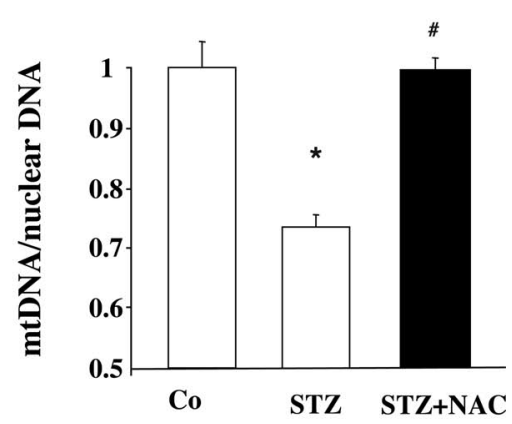

D
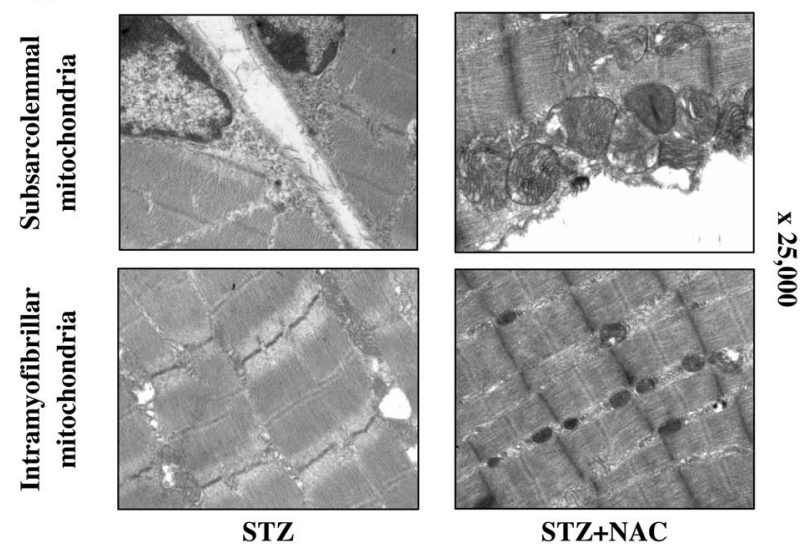

Figure 6

Antioxidant treatment restores mitochondrial alterations in STZ mice. (A) Plasma $\mathrm{H}_{2} \mathrm{O}_{2}$ levels in Co and STZ mice treated or not with NAC (10 mM in drinking water). (B) Immunoblots showing total protein carbonylation in the gastrocnemius muscle of control, STZ, and NAC-treated STZ mice. (C) mtDNA copy number was calculated as the ratio of COX1 to cyclophilin A DNA levels, determined by real-time PCR, in the skeletal muscle of control, STZ, and NAC-treated STZ mice. Note that the $y$ axis scale is between 0.5 and 1 . Results were normalized by the mean value of the control condition set to 1 unit. ${ }^{*} P<0.01$ vs. control; $P P<0.05$ vs. STZ. (D) Transmission electron microscopy images (original magnification, $\times 25,000)$ of subsarcolemmal and intermyofibrillar mitochondria from the gastrocnemius muscle of STZ and NAC-treated STZ mice.

This working hypothesis assumes that the intake of a highenergy diet for a prolonged period of time might be an initiating factor for the generation of ROS locally in skeletal muscle. In agreement with this assumption, skeletal muscle mitochondria are generally not altered in genetic models of obesity. Indeed, we observed no mitochondrial dysfunction in the muscle of KKAy mice. Similarly, a recent report indicates that mitochondria are not altered in the skeletal muscle of genetically obese $(o b / o b)$ and diabetic $(d b / d b)$ mice (31). However, it cannot be excluded that leptin receptor mutation and decreased leptin signalling could play a protective role in these models, because leptin has been shown to increase the production of ROS (32).

In summary, the present study demonstrates that mitochondrial dysfunction is not an early event in the development of insulin resistance in diabetic mice, but rather is a complication of hyperglycemia- and hyperlipidemia-induced ROS production in skeletal muscle. If a similar mechanism occurs in humans, our data suggest that mitochondrial dysfunction, as observed in the skeletal muscle of type 2 diabetic and prediabetic patients (11, $12,18)$, is probably not the initial event that triggers decreased oxidative capacity, lipid accumulation, and inhibition of insu- lin action. Under such conditions, increased oxidative stress in the skeletal muscle might be a unifying mechanism promoting mitochondrial alterations, lipid accumulation, and insulin resistance. Because increased ROS levels also play an important role in altered insulin secretion by the pancreas (33), oxidative stress might contribute to the 2 prominent features of type 2 diabetes: insulin resistance and pancreatic $\beta$ cell dysfunction. Therefore, therapeutic strategies to limit mitochondrial radical production and to counteract their damaging effects may provide a useful complement to conventional therapies designed to normalize blood glucose and lipids.

\section{Methods}

Animals. Male C57BL/6 mice at 4 weeks (diet protocol) and 10 weeks (STZ protocol) of age were purchased from Harlan. Male C57BL/6J and KKAy mice at 9 weeks of age were purchased from the Jackson Laboratory. Animals were housed in the common animal center from Laennec faculty medicine (IFR62, Lyon) at $22^{\circ} \mathrm{C}$ and with a 12 -h light/dark cycle. Animal procedures were conducted in accordance with the institutional guidelines for the care and use of laboratory animals. After 1 week of acclimatization, mice in the diet protocol were divided into 2 groups: 1 with free 
A

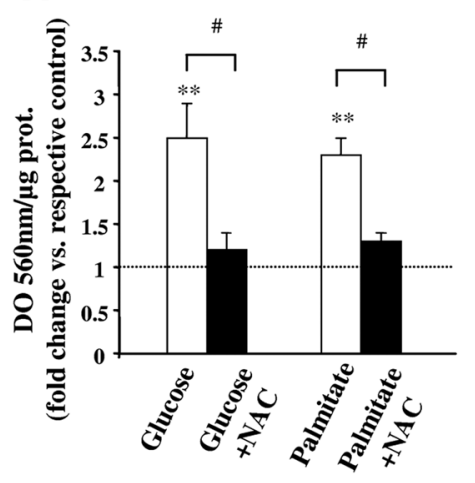

B

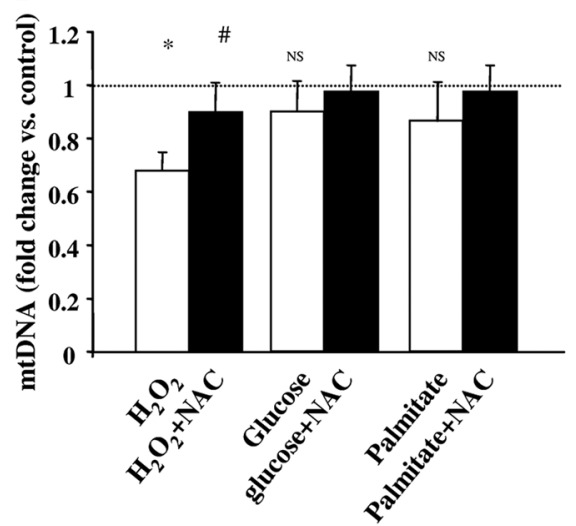

D
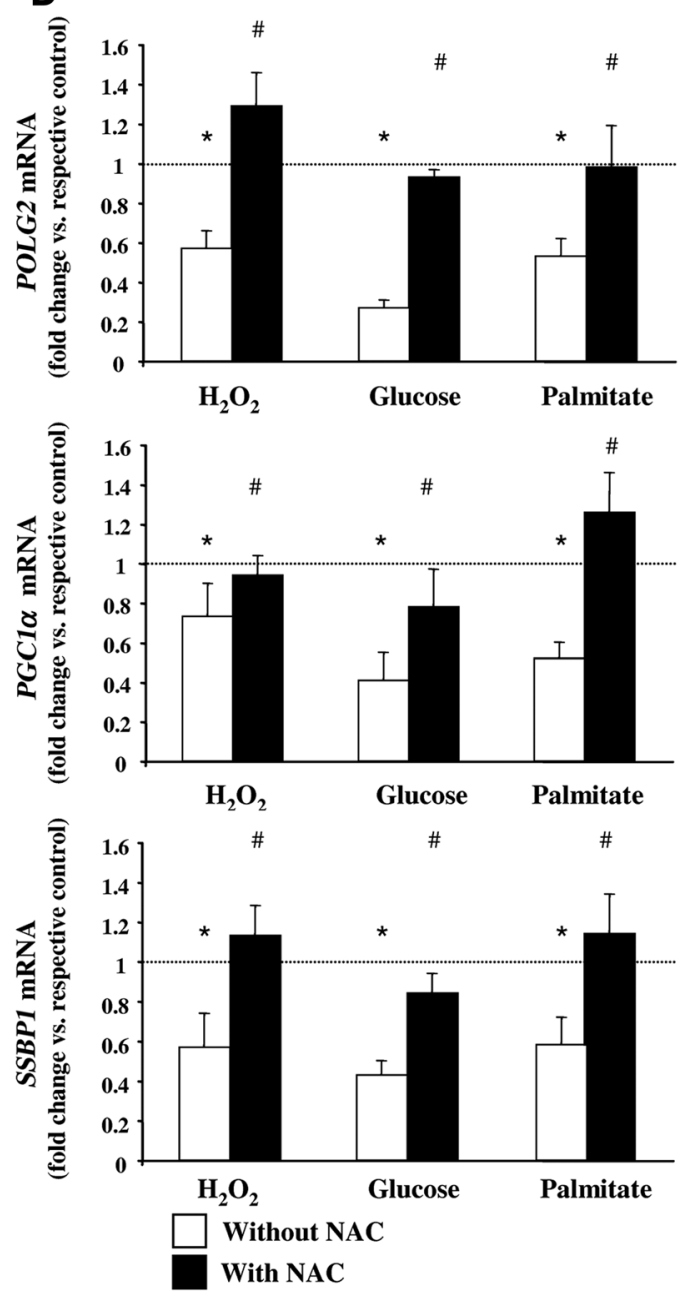

Figure 7

ROS-induced mitochondrial alterations in $\mathrm{C}_{2} \mathrm{C}_{12}$ muscle cells. (A) ROS production, measured by NBT reduction, in differentiated $\mathrm{C}_{2} \mathrm{C}_{12}$ myotubes incubated with glucose $(25 \mathrm{mM})$ or palmitate $(200 \mu \mathrm{M})$ in the presence or absence of NAC $(10 \mathrm{mM})$ for 96 hours $(n=4)$. Data are expressed relative to the respective control (dotted line). (B) Effect of $\mathrm{H}_{2} \mathrm{O}_{2}(0.1 \mathrm{mM})$, glucose $(25 \mathrm{mM})$, and palmitate $(200 \mu \mathrm{M})$ on mtDNA levels in differentiated $\mathrm{C}_{2} \mathrm{C}_{12}$ myotubes. Myotubes were treated for 96 hours in the presence or absence of $10 \mathrm{mM} \mathrm{NAC}(n=4)$. Data are expressed relative to the control condition (dotted line). (C) CS activity measured in total lysates of myotubes treated for 96 hours with $\mathrm{H}_{2} \mathrm{O}_{2}(0.1 \mathrm{mM})$, glucose $(25 \mathrm{mM})$, or palmitate $(200 \mu \mathrm{M})$ in the presence or absence of NAC $(10 \mathrm{mM})(n=4)$. (D) mRNA levels of POLG2, SSBP1, and PGC1 $\alpha$ genes determined by quantitative RT-PCR in $\mathrm{H}_{2} \mathrm{O}_{2}$-, glucose-, or palmitate-treated myotubes in the presence or absence of NAC for 96 hours $(n=4)$. All results are expressed as fold change relative to the values of untreated cells set to 1 unit (dotted line). ${ }^{*} P<0.05,{ }^{*} P<0.01$ vs. respective control; $\# P<0.05$ vs. without NAC.

access to a standard chow diet (SD, 57\% carbohydrate, 5\% fat, and $18 \%$ protein; Harlan) and 1 with free access to a pelleted HFHSD (36\% fat, $35 \%$ carbohydrate [50\% sucrose], and $19.8 \%$ protein; TD 99249; Harlan). Animals were studied after 4 and 16 weeks of feeding. At the end of the protocols, blood was withdrawn in a fed state from the orbital sinus of anesthetized animals with a heparinized microcapillary tubes. Then, animals were sacrificed by cervical dislocation, and gastrocnemius muscles were rapidly excised and frozen in liquid nitrogen.

For the STZ study, 10-week-old C57BL/6 mice were given an intraperitoneal dose of STZ dissolved in sodium citrate buffer $(100 \mathrm{mg} / \mathrm{kg}$ body weight; Sigma-Aldrich) daily for 3 consecutive days. Glucose levels were monitored daily, and mice were studied when they achieved fed glucose levels of $>500 \mathrm{mg} / \mathrm{dl}$ for 3 consecutive days (day 11). One group was treated with insulin (Insulatard, $3 \mathrm{mU}$ ) and another group with phlorizin $(0.2 \mathrm{~g} / \mathrm{kg}$ ); both substances were given twice daily at 8-hour intervals. Control groups for each treatment were infused with the respective vehicle. Twenty-four hours after the first injection of insulin or phlorizin, animals were sacrificed, and gastrocnemius muscles were removed and frozen. Another group of STZ mice was treated with a general antioxidant, NAC $(10 \mathrm{mM}$ in drinking water), starting from the seventh day after the first injection of STZ. Mice were sacrificed after 5 days of NAC treatment, and blood and samples were obtained as described above.

Measurement of metabolites and hormones. Blood glucose levels were measured using a glucometer (Roche Diagnostics). Serum levels of insulin (Linco Research) and leptin (BioVendor) were determined with murine ELISA kits. Total serum triglycerides (Biomérieux) and FFAs (Roche Diagnostics) were assayed using enzymatic methods. Plasma $\mathrm{H}_{2} \mathrm{O}_{2}$ levels were measured using an Amplex Red hydrogen peroxide assay kit (Invitrogen). 
Transmission electron microscopy. Gastrocnemius muscle was cut into small pieces and fixed in $2 \%$ glutaraldehyde for 2 hours at $4{ }^{\circ} \mathrm{C}$, postfixed in $1 \%$ Osmium tetroxide for 1 hour at $4^{\circ} \mathrm{C}$, dehydrated, and embedded in Epon at either a longitudinal or transverse orientation. The tissue was then cut using an RMC/MTX ultramicrotome (Elexience), and ultrathin sections $(60-80 \mathrm{~nm})$ were mounted on copper grids, contrasted with $8 \%$ uranyl acetate and lead citrate, and observed with a Jeol 1200 EX transmission electron microscope (Jeol LTD) equipped with a MegaView II high-resolution transmission electron microscopy camera. The analysis was performed with Soft Imaging System (Eloïse SARL). The selection of oxidative fibers was based on the size of fibers and the amount of mitochondria.

Mitochondrial DNA analysis. Total DNA was extracted from muscle using phenol/chloroform/isoamyl alcohol (25:24:1) followed by ethanol precipitation. The content of mtDNA was calculated using real-time quantitative PCR by measuring the threshold cycle ratio $(\Delta \mathrm{Ct})$ of a mitochondrialencoded gene (COX1, forward 5'-ACTATACTACTACTAACAGACCG-3', reverse 5'-GGTTCTTTTTTTCCGGAGTA-3') versus a nuclear-encoded gene (cyclophilin A, forward 5'-ACACGCCATAATGGCACTGG-3', reverse 5'-CAGTCTTGGCAGTGCAGAT-3').

Real-time quantitative RT-PCR analysis. Total RNA was extracted with the TRIzOL Reagent (Invitrogen). The level of target mRNAs was measured by RT followed by real-time PCR using a LightCycler (Roche). A standard curve was systematically generated with 8 different amounts of purified target cDNA, and each assay was performed in duplicate (34). Briefly, first-strand cDNAs were first synthesised from $500 \mathrm{ng}$ total RNA in the presence of $100 \mathrm{U}$ Superscript II (Invitrogen) using random hexamers and oilgo(dT) primers (Promega). The real-time RT-PCR was performed in a final concentration of $20 \mu \mathrm{l}$ containing $5 \mu \mathrm{l}$ RT reaction medium at 60 -fold dilution, $15 \mu \mathrm{l}$ reaction buffer from the FastStart DNA Master SYBR Green kit (Roche), and 10.5 pmol specific forward and reverse primers (Eurobio). Primer sequences are shown in Supplemental Table 2.

Measurement of mitochondrial respiration on skinned fiber preparation. Mitochondrial respiration was studied in saponin-skinned fibers (35). Fiber bundles were mechanically separated with tongs and permeabilized with saponin (60 mg/l, 20 minutes). Bundles were then washed 3 times for 10 minutes to remove ADP, creatine phosphate, soluble enzymes, and metabolites. Fiber respiration rates were measured at $25^{\circ} \mathrm{C}$ using an oxygraph system (Hansatech Instruments). Different substrates were used as follows: $5 \mathrm{mM}$ glutamate plus $2 \mathrm{mM}$ malate as complex 1 substrates, $5 \mathrm{mM}$ succinate plus $2.2 \mu \mathrm{M}$ rotenone as complex 2 substrates with inhibition of complex 1 by rotenone, octanoyl-carnitine $(110 \mu \mathrm{M})$ or palmitoyl-carnitine $(55 \mu \mathrm{M})$ in the presence of $1 \mathrm{mM}$ malate as $\beta$-oxidation substrates. State 3 was measured in the presence of respiratory substrates after the addition of $1 \mathrm{mM} \mathrm{ADP}$, and state 4 was measured after the addition of $60 \mu \mathrm{M}$ atractyloside - a potent inhibitor of the ATP/ADP carrier. State 4 was considered the control state of respiration. Finally, fibers were dried for 24 hours at $100^{\circ} \mathrm{C}$ and weighed. Respiration rates were expressed as nanoatoms $\mathrm{O} /(\mathrm{min} . \mathrm{mg}$ dried fiber). The respiratory control ratio was calculated by dividing state 3 by state 4 respiration rates.

Mitochondria isolation. Muscle was thawed in isolation buffer $(210 \mathrm{mM}$ mannitol, $70 \mathrm{mM}$ saccharose, $50 \mathrm{mM}$ Tris, $10 \mathrm{mM}$ EDTA, and 0.5\% BSA; $\mathrm{pH}$ 7.4) and cut in small pieces. It was then digested for 15 minutes with trypsin, under agitation, and washed 2 times with the isolation buffer. After each wash, the tissue was centrifuged at $4{ }^{\circ} \mathrm{C}$ for 2 minutes at $70 \mathrm{~g}$. The tissue was homogenized with a conical glass grinder (VWR International) in $1 \mathrm{ml}$ isolation buffer. The homogenate was centrifuged at $4^{\circ} \mathrm{C}$ for 10 minutes at $820 \mathrm{~g}$. The supernatant was then centrifuged at $4^{\circ} \mathrm{C}$ for 20 minutes at $6,800 \mathrm{~g}$. The pellet was resuspended in $1 \mathrm{ml}$ of suspension buffer (225 mM mannitol, $75 \mathrm{mM}$ saccharose, $10 \mathrm{mM}$ Tris, and $0.1 \mathrm{mM}$ EDTA; $\mathrm{pH} 7.4$ ) and centrifuged at $4^{\circ} \mathrm{C}$ for 10 minutes at $820 \mathrm{~g}$. The mitochondria were then pelleted by centrifuging the supernatant at $4^{\circ} \mathrm{C}$ for 20 minutes at $6,800 \mathrm{~g}$ and were resuspended in $50 \mu \mathrm{l}$ of the same buffer. Western blot analysis was used to determine the cytochrome $c$ (1:100; Santa Cruz) content in cytosolic and mitochondrial fractions.

Measurement of enzyme activities. CS activity was measured spectrophotometrically (36) in purified mitochondria from gastrocnemius muscle. For the measurement of caspase 3 activity, gastrocnemius muscle was lysed in ice-cold lysis buffer (1\% NP-40, $20 \mathrm{mM}$ Tris- $\mathrm{HCl}, 138 \mathrm{mM} \mathrm{NaCl}$, $2.7 \mathrm{mM} \mathrm{KCl}, 1 \mathrm{mM} \mathrm{MgCl}$, $5 \%$ glycerol; $\mathrm{pH} 8.0$ ) and supplemented with $5 \mathrm{mM}$ EDTA $\left(1 \mathrm{mM} \mathrm{Na}_{3} \mathrm{VO}_{4}, 20 \mathrm{mM} \mathrm{NaF}, 1 \mathrm{mM} \mathrm{DTT}\right.$, and protease inhibitors). After centrifugation at $10,000 \mathrm{~g}$ for 10 minutes at $4{ }^{\circ} \mathrm{C}$, aliquots of supernatant $(100 \mu \mathrm{g})$ were incubated with $10 \mu \mathrm{l}$ DEVD-pNA for 1 hour at $37^{\circ} \mathrm{C}$ according to the instructions of the manufacturer (Clinisciences). The pNA light emission was quantified using a spectrophotometer at $400 \mathrm{~nm}$.

Protein carbonylation. The Oxyblot Oxidized Protein Detection Kit was purchased from Chemicon. The carbonyl groups in the protein side chains were derivatized to DNP-hydrazone by reaction with DNPH following the manufacturer's instructions. After the derivatization of the protein sample, 1-dimensional electrophoresis was carried out on a $10 \%$ SDS-PAGE gel. Proteins were transferred to PVDF membranes. After incubation with anti-DNP antibody, the blot was developed using a chemiluminescence detection system.

Muscle cell culture. $\mathrm{C}_{2} \mathrm{C}_{12}$ myoblasts were grown to confluence in DMEM supplemented with $10 \%$ FCS. They were induced to differentiate into myotubes by switching to DMEM containing $2 \%$ horse serum. Primary cultures of human skeletal muscle cells were initiated from satellite cells of quadriceps samples obtained from 3 male organ donors (age, $27 \pm 7$ years; body mass index, $23 \pm 1.7 \mathrm{~kg} / \mathrm{m}^{2}$ ). Differentiated myotubes were prepared according to the procedure previously described in detail (37). Myotubes were then cultured for 96 hours, with or without $\mathrm{H}_{2} \mathrm{O}_{2}(100 \mu \mathrm{M})$, with mannitol $(25 \mathrm{mM})$ or glucose $(25 \mathrm{mM})$, and with BSA $(1 \%)$ or palmitic acid $(200 \mu \mathrm{M})$, in the presence or absence of NAC $(10 \mathrm{mM})$.

ROS production in $\mathrm{C}_{2} \mathrm{C}_{12}$ cells. ROS production was detected using the nitroblue tetrazolium (NBT; Sigma-Aldrich) assay. NBT is reduced by ROS to a dark-blue insoluble form of NBT called formazan. After treatment, myotubes were incubated for 90 minutes in DMEM containing $0.2 \%$ NBT. Formazan was dissolved in 50\% acetic acid, and the absorbance was determined at $560 \mathrm{~nm}$. Optical density values were normalized by protein levels.

Statistics. To analyze the difference between control and experimental groups, 2-tailed Student's $t$ test was used. A $P$ value less than 0.05 was considered to be significant.

\section{Acknowledgments}

We thank the imagery center of Laennec Faculty and the IFR62 for access to the platforms. This work was supported by grants from INSERM, the French National Research Agency (ANR05-PCOD-012), and the French National Program on Diabetes Research (to J. Rieusset). C. Bonnard received fellowships from the Rhônes-Alpes region.

Received for publication May 4, 2007, and accepted in revised form October 24, 2007.

Address correspondence to: Jennifer Rieusset, INSERM U870, Faculté de Médecine Lyon Sud, 165 Chemin du Grand Revoyet, 69600 Oullins. Phone: 33-4-26-23-59-20; Fax: 33-4-26-23-59-16; E-mail: jennifer.rieusset@univ-lyon1.fr. 
1. Krebs, M., and Roden, M. 2004. Nutrient-induced insulin resistance in human skeletal muscle. Curr Med. Chem. 11:901-908.

2. Perseghin, G., et al. 1999. Intramyocellular triglyceride content is a determinant of in vivo insulin resistance in humans: a $1 \mathrm{H}-13 \mathrm{C}$ nuclear magnetic resonance spectroscopy assessment in offspring of type 2 diabetic parents. Diabetes. 48:1600-1606

3. Krssak, M., et al. 1999. Intramyocellular lipid concentrations are correlated with insulin sensitivity in humans: a $1 \mathrm{H}$ NMR spectroscopy study. Diabetologia. 42:113-116.

4. Hegarty, B.D., Furler, S.M., Ye, J., Cooney, G.J., and Kraegen, E.W. 2003. The role of intramuscular lipid in insulin resistance. Acta. Physiol. Scand. 178:373-383

5. Schmitz-Peiffer, C., Craig, D.L., and Biden, T.J. 1999. Ceramide generation is sufficient to account for the inhibition of the insulin-stimulated PKB pathway in $\mathrm{C} 2 \mathrm{C} 12$ skeletal muscle cells pretreated with palmitate. J. Biol. Chem. 274:24202-24210.

6. Yu, C., et al. 2002. Mechanism by which fatty acids inhibit insulin activation of insulin receptor substrate-1 (IRS-1)-associated phosphatidylinositol 3-kinase activity in muscle. J. Biol. Chem 277:50230-50236.

7. Bonen, A., et al. 2004. Triacylglycerol accumulation in human obesity and type 2 diabetes is associated with increased rates of skeletal muscle fatty acid transport and increased sarcolemmal FAT/CD36. FASEB J. 18:1144-1146.

8. Hegarty, B.D., Cooney, G.J., Kraegen, E.W., and Furler, S.M. 2002. Increased efficiency of fatty acid uptake contributes to lipid accumulation in skeletal muscle of high fat-fed insulin-resistant rats. Diabetes. 51:1477-1484.

9. Lowell, B.B., and Shulman, G.I. 2005. Mitochondrial dysfunction and type 2 diabetes. Science. 307:384-387.

10. Simoneau, J.A., and Kelley, D.E. 1997. Altered glycolytic and oxidative capacities of skeletal muscle contribute to insulin resistance in NIDDM. J. Appl. Physiol. 83:166-171.

11. Petersen, K.F., et al. 2004. Impaired mitochondrial activity in the insulin-resistant offspring of patients with type 2 diabetes. N. Engl. J. Med. 350:664-671.

12. Kelley, D.E., He, J., Menshikova, E.V., and Ritov, V.B. 2002. Dysfunction of mitochondria in human skeletal muscle in type 2 diabetes. Diabetes. 51:2944-2950.

13. Mootha, V.K., et al. 2003. PGC-1alpha-responsive genes involved in oxidative phosphorylation are coordinately downregulated in human diabetes. Nat. Genet. 34:267-273.

14. Patti, M.E., et al. 2003. Coordinated reduction of genes of oxidative metabolism in humans with insulin resistance and diabetes: potential role of PGC1 and NRF1. Proc. Natl. Acad. Sci. U. S. A. 100:8466-8471.

15. Sparks, L.M., et al. 2005. A high-fat diet coordinately downregulates genes required for mitochondrial oxidative phosphorylation in skeletal muscle. Diabetes. 54:1926-1933.

16. Graziewicz, M.A., Longley, M.J., and Copeland, W.C. 2006. DNA polymerase gamma in mitochondrial DNA replication and repair. Chem. Rev. 106:383-405

17. Schrauwen, P., and Hesselink, M.K.C. 2004. The role of uncoupling protein 3 in fatty acid metabolism: protection against lipotoxicity? Proc. Nutr. Soc 63:287-292.

18. McGarry, J.D. 2002. Banting lecture 2001: dysregulation of fatty acid metabolism in the etiology of type 2 diabetes. Diabetes. 51:7-18.

19. Morino, K., et al. 2005. Reduced mitochondrial density and increased IRS-1 serine phosphorylation in muscle of insulin-resistant offspring of type 2 diabetic parents. J. Clin. Invest. 115:3587-3593.

20. Puigserver, P., and Spiegelman, B.M. 2003. Peroxisome proliferator-activated receptor-gamma coactivator 1 alpha (PGC-1 alpha): transcriptional coactivator and metabolic regulator. Endocr. Rev. 24:78-90.

21. Debard, C., et al. 2004. Expression of key genes of fatty acid oxidation, including adiponectin receptors, in skeletal muscle of Type 2 diabetic patients. Diabetologia. 47:917-925.

22. Bach, D., et al. 2003. Mitofusin-2 determines mitochondrial network architecture and mitochondrial metabolism. A novel regulatory mechanism altered in obesity. J. Biol. Chem. 278:17190-17197.

23. Nishikawa, T., et al. 2000. Normalizing mitochondrial superoxide production blocks three pathways of hyperglycaemic damage. Nature. 404:787-790.

24. Furukawa, S., et al. 2004. Increased oxidative stress in obesity and its impact on metabolic syndrome.
J. Clin. Invest. 114:1752-1761.

25. Russell, J.W., et al. 2002. High glucose-induced oxidative stress and mitochondrial dysfunction in neurons. FASEB J. 16:1738-1748.

26. Graziewicz, M.A., Day, B.J., and Copeland, W.C. 2002. The mitochondrial DNA polymerase as a target of oxidative damage. Nucleic Acids Res. 30:2817-2824

27. Evans, J.L., Maddux, B.A., and Goldfine, I.D. 2005. The molecular basis for oxidative stressinduced insulin resistance. Antioxid. Redox. Signal. 7:1040-1052.

28. Schrauwen, P. 2007. High-fat diet, muscular lipotoxicity and insulin resistance. Proc. Nutr. Soc. 66:33-41.

29. Lagouge, M., et al. 2007. Resveratrol improves mitochondrial function and protects against metabolic disease by activating SIRT1 and PGC-1alpha. Cell. 127:1109-1122.

30. Gerhart-Hines, Z., et al. 2007. Metabolic control of muscle mitochondrial function and fatty acid oxidation through SIRT1/PGC-1alpha. EMBO J. 26:1913-1923.

31. Choo, H.J., et al. 2006. Mitochondria are impaired in the adipocytes of type 2 diabetic mice. Diabetologia. 49:784-791.

32. Kutlu, S., et al. 2005. Exogenous leptin increases lipid peroxidation in the mouse brain. Tohoku J. Exp. Med. 206:233-236.

33. Evans, J.L., Goldfine, I.D., Maddux, B.A., and Grodsky, G.M. 2002. Oxidative stress and stress-activated signaling pathways: a unifying hypothesis of type 2 diabetes. Endocr. Rev. 23:599-622.

34. Rome, S., et al. 2003. Microarray profiling of human skeletal muscle reveals that insulin regulates approximately 800 genes during a hyperinsulinemic clamp. J. Biol. Chem. 278:18063-18068.

35. Saks, V.A., et al. 1998. Permeabilized cell and skinned fiber techniques in studies of mitochondrial function in vivo. Mol. Cell. Biochem. 184:81-100.

36. Shepherd, D., and Garland, P.B. 1969. The kinetic properties of citrate synthase from rat liver mitochondria. Biochem. J. 114:597-610.

37. Rieusset, J., et al. 2004. Suppressor of cytokine signaling 3 expression and insulin resistance in skeletal muscle of obese and type 2 diabetic patients. Diabetes. 53:2232-2241. 